\title{
Epidémiologie des nématodes gastro-intestinaux des bovins dans la région centre de la Côte d'Ivoire
}

\author{
C. Komoin-Oka ${ }^{1}$ J. Zinsstag ${ }^{2}$ F. Fofana ${ }^{1}$ \\ A. N'Depo ${ }^{1}$ V.S. Pandey ${ }^{3 *}$
}

\section{Mots-clés}

Bovin N'Dama - Helminthe - Nematoda - Epidémiologie - Côte d'Ivoire.

\section{Résumé}

De février à décembre 1997, six bovins de race N'Dama, provenant de la zone centre de savane humide de la Côte d'Ivoire, ont été autopsiés chaque mois. Quatorze espèces ou genres de parasites ont été mis en évidence : 8 nématodes, 2 trématodes, 1 cestode et 3 protozoaires. Tous les animaux étaient infestés par au moins l'une des espèces suivantes de nématodes gastrointestinaux : Haemonchus sp. (95 p. 100), Cooperia punctata (50 p. 100), O esophagostomum radiatum (35,5 p. 100), Trichostrongylus axei (30 p. 100), Cooperia pectinata $(8,1$ p. 100$)$ et Trichuris sp. (1,6 p. 100). Setaria labiatopapillosa a été rencontrée chez 30,6 p. 100 des animaux, des microfilaires chez 24,1 p. 100 , des paramphistomes chez 80,6 p. 100 , et M oniezia sp. chez 15 p. 100. Des trypanosomes et Babesia spp. ont été trouvés chez respectivement 11,2 et 3,6 p. 100 des animaux. Les excrétions d'œufs et les intensités parasitaires des nématodes gastro-intestinaux ont montré des fluctuations avec des pics en mai et en septembre-octobre, périodes correspondant aux saisons des pluies. Les animaux âgés de 1-3 ans avaient des intensités parasitaires plus élevées que ceux âgés de plus de 3 ans $(P<0,05)$. Aucune hypobiose larvaire n'a été détectée. En dépit de conditions climatiques humides favorables, les intensités parasitaires de ces bovins de race N'Dama, provenant de systèmes d'élevage de type extensif, étaient relativement faibles. Des traitements anthelminthiques ne seraient alors justifiés que chez les jeunes animaux.

\section{INTRO DUCTION}

Au cours de ces dernières années, les auteurs se sont de plus en plus intéressés au bovin de race N'Dama de l'Afrique de l'Ouest, étant donné sa trypanotolérance et sa résistance aux tiques et aux maladies transmises par les tiques $(6,8)$. Le N'Dama serait également plus résistant que d'autres races bovines aux infections par les strongles (7). Des études menées en Gambie $(3,14,15)$, au Sénégal (10) et en Guinée (1) montrent que le parasitisme dû aux strongles gastro-intestinaux peut néanmoins être important, même chez le bovin de race N'Dama.

1. Lanada, Laboratoire central de pathologie animale de Bingerville, BP 206 Bingerville, Côte d'Ivoire

2. Institut tropical suisse, CH-4002 Bâle, Suisse ; Centre suisse de recherches scientifiques, BP 1303 Abidjan 01, Côte d'Ivoire

3. Institut de médecine tropicale, Nationalestraat 155, B-2000 Antwerpen, Belgique

* Auteur pour la correspondance

Tél. : +32-2-251 8436 ; Fax : +32-2-251 8436 ; E-mail : vpandey@itg.be
En Côte d'Ivoire, le développement de l'élevage est une priorité. Dans les régions sud et centre du pays où le climat est humide, l'élevage d'animaux trypanotolérants est encouragé. Ces animaux sont élevés sur des pâturages naturels, dans un système de type extensif. Compte tenu de l'importance reconnue aux helminthes gastro-intestinaux, les services vétérinaires recommandent trois traitements anthelminthiques par an (information non publiée). Cependant, il n'y a pas de publications faisant état de l'épidémiologie et des variations saisonnières du parasitisme. L'objectif du présent travail a été de mener une étude épidémiologique par l'examen régulier d'animaux abattus dans l'un des abattoirs de la région centre de la Côte d'Ivoire.

\section{MATERIEL ET METHODES}

\section{Zone de l'étude}

Les animaux examinés provenaient de la zone centre de savane de la Côte d'Ivoire (6-7 ${ }^{\circ}$ de latitude N, 5- $6^{\circ}$ de longitude O), région caractérisée par un climat tropical humide, aux températures 
comprises entre 19 et $34{ }^{\circ} \mathrm{C}$ et à la pluviométrie annuelle variant entre 1100 et $1600 \mathrm{~mm}$, avec des pics en mai-juin et en septembre-octobre. Durant la période de l'étude, les données climatiques ont été obtenues auprès de la Société d'exploitation et de développement aéroportuaire, aéronautique et météorologique (Sodexam) d'Abidjan.

\section{Les animaux}

De février à décembre 1997, cinq à six bovins de race N'Dama, abattus à l'abattoir municipal de Toumodi, ont été examinés tous les mois. Les critères de sélection de ces animaux étaient leur lieu d'origine (la zone de l'étude) et l'absence de traitement antiparasitaire depuis au moins six mois. Au total, 62 animaux ont ainsi été examinés, constitués de 16 mâles et de 46 femelles, de 22 bovins âgés de 1 à 3 ans et de 40 âgés de plus de 3 ans.

\section{Méthodes parasitologiques}

Avant l'abattage, du sang était prélevé à l'oreille et des selles étaient récoltées dans le rectum des animaux. Le sang était examiné pour la recherche d'hémoparasites, selon la technique de centrifugation en tube à hématocrite de Murray et coll. (9) et après coloration de frottis et gouttes épaisses au Giemsa. Les fèces étaient examinées selon la méthode de McMaster modifiée d'après Gordon et Whitlock (2) et par sédimentation à l'eau du robinet et flottation dans une solution sursaturée de $\mathrm{NaCl}(\mathrm{d}=1,20)$.

Après éviscération, les poumons, le foie, le rumen, le cœur et la carcasse étaient inspectés. Les veines mésentériques étaient examinées pour la recherche de schistosomes. L'abomasum, l'intestin grêle et le gros intestin étaient séparés. Chaque portion était ouverte dans le sens de la longueur et son contenu était lavé et passé à travers un tamis de $200 \mu$. Les résidus du tamis étaient complétés avec 51 d'eau et un échantillon de $200 \mathrm{ml}$ était collecté. Les parasites étaient récoltés et identifiés au microscope.

Les nodules de l'intestin grêle, du côlon et du cæcum étaient comptés. La muqueuse de la moitié de l'abomasum et celle de la portion du cæcum allant jusqu'à la valvule iléo-cæcale étaient grattées et mises à digérer (dans $10 \mathrm{~g}$ de pepsine à $1200 \mathrm{UI} / \mathrm{g}+8,5 \mathrm{~g}$ de $\mathrm{NaCl}+16 \mathrm{ml}$ de $\mathrm{HCl}$ à $37 \mathrm{p} .100+1000 \mathrm{ml}$ d'eau distillée) à $37{ }^{\circ} \mathrm{C}$ pendant $12 \mathrm{~h}$ pour la recherche de larves (5).

\section{Analyse statistique}

La saisie et la vérification des données ont été opérées à l'aide du programme EPI-INFO (version 5.0). L'analyse statistique a été effectuée avec le programme SAS (Statistical Analysis Systems, Cary, USA) après transformation logarithmique des données brutes. Des comparaisons statistiques ont été faites à l'aide du test de Wilcoxon ou par l'analyse de variance. Le seuil de signification a été fixé à $\mathrm{p}<5$ p. 100.

\section{RESU LTATS}

Les moyennes mensuelles des températures et des précipitations sont présentées dans la figure 1. Durant l'année de l'étude, la pluviométrie totale a été de $1179 \mathrm{~mm}$; les températures moyennes mensuelles ont oscillé entre 24,5 et $27,7^{\circ} \mathrm{C}$, et l'humidité relative a été en moyenne de 72,3 p. 100.

Un total de 14 espèces ou genres de parasites a été mis en évidence : 8 nématodes, 2 trématodes, 1 cestode et 3 protozoaires (tableau I). Tous les animaux étaient porteurs au moins de deux espèces parasitaires, sauf trois d'entre eux, infestés par une seule espèce, Haemonchus sp. Haemonchus était prédominant par sa prévalence (95 p. 100) et son intensité moyenne (900 vers). Il représentait 96 p. 100 de la population abomasale. Aucune larve n'a été observée dans le digestat abomasal. Dans l'intestin grêle et le gros intestin, Cooperia punctata et Oesophagostomum radiatum étaient respectivement les espèces les plus importantes. Environ 90 p. 100 des animaux avaient 10 à 120 nodules dans l'intestin. Aucune larve n'a été retrouvée après digestion de la muqueuse cæcale.

L'intensité de la totalité des nématodes gastro-intestinaux a varié entre 25 et 5203 parasites ; 63 p. 100 des animaux avaient moins de 1000 nématodes, 28 p. 100 en avaient entre 1000 et 3000 , et 9 p. 100 en avaient plus de 3000 .

L'intensité parasitaire abomasale était comprise entre 0 et 5151 nématodes, avec 5 p. 100 des animaux n'hébergeant aucun parasite dans l'abomasum, 61,7 p. 100 en hébergeant moins de 1000 , 28,3 p. 100 entre 1000 et 3000 , et 5 p. 100 plus de 3000 . Le nombre de nématodes dans l'intestin grêle a oscillé entre 0 et 2183 nématodes, avec 50 p. 100 des animaux n'en ayant aucun et un seul animal en ayant plus de 2000 . La plupart des animaux (64,5 p. 100) n'avaient pas de nématodes dans le gros intestin. Un seul en avait 1200 et 33,9 p. 100 avaient moins de 1000 $O$. radiatum.

L'évolution des différentes espèces de nématodes a connu des variations au cours de l'année (figure 2). Des pics parasitaires ont été observés au mois d'octobre pour Haemonchus sp., en mai et en décembre pour $T$. axei, en mars, en mai et en décembre pour C. punctata, et en mai pour $O$. radiatum. L'intensité totale des nématodes gastro-intestinaux a augmenté progressivement pour atteindre un pic en juillet, avant de baisser puis de connaître un second pic en octobre (figure 3). Ces fluctuations n'étaient statistiquement significatives que pour $C$. punctata $(\mathrm{p}=0,02)$.

Des œufs de strongles ont été détectés dans les fèces de 70 p. 100 des animaux avec un opg moyen de 305 (extrêmes : 50-750). Ces excrétions d'œufs ont connu des variations au cours de l'année ( $\mathrm{p}<0,01$ ), avec un pic principal en septembre (figure 4). Des œufs de cestodes étaient présents chez 12 p. 100 des animaux et des oocystes de coccidies chez 9,3 p. 100.

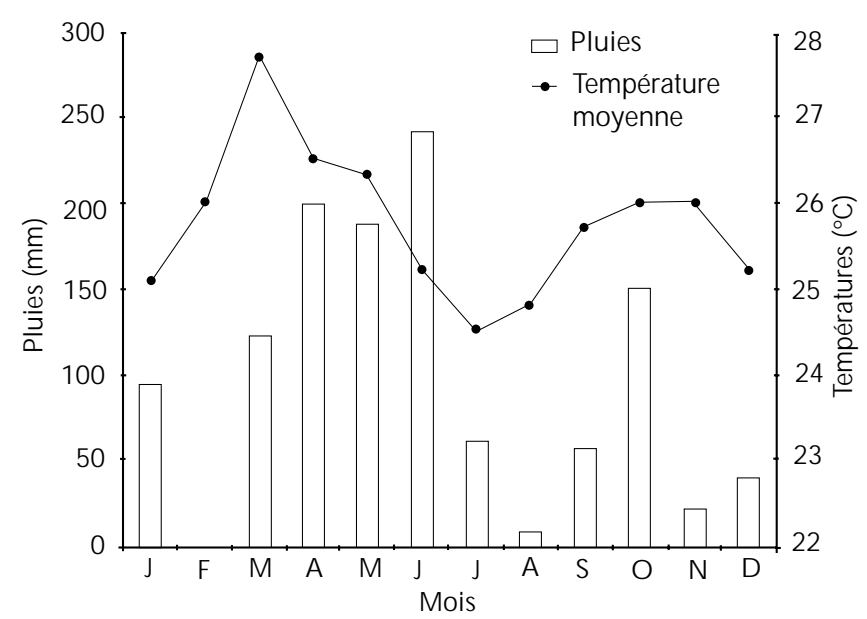

Figure 1 : températures et précipitations moyennes mensuelles dans la zone centre de savane humide de la Côte d'Ivoire de janvier à décembre 1997. 


\section{Tableau I}

Inventaire, prévalence, intensités (moyenne et extrêmes) des parasites des bovins ( $n=62$ ) de la zone centre de savane humide de la Côte d'Ivoire

\begin{tabular}{|c|c|c|c|}
\hline \multirow[t]{2}{*}{ Parasites } & \multirow[t]{2}{*}{ Prévalence (\%) } & \multicolumn{2}{|c|}{ Intensités des positifs } \\
\hline & & $\begin{array}{c}\text { Moyenne } \\
\text { (Nb. de parasites) }\end{array}$ & $\begin{array}{c}\text { Extrêmes } \\
\text { (Nb. de parasites) }\end{array}$ \\
\hline \multicolumn{4}{|l|}{ Rumen } \\
\hline Paramphistomes & 80,6 & & \\
\hline \multicolumn{4}{|l|}{ Caillette } \\
\hline H aemonchus sp. & 95 & 900 & $25-5151$ \\
\hline Trichostrongylus axei & 30 & 115 & $9-650$ \\
\hline \multicolumn{4}{|l|}{ Intestin grêle } \\
\hline Cooperia pectinata & 8,1 & 73 & $4-175$ \\
\hline Cooperia punctata & 50 & 272 & $1-2008$ \\
\hline Moniezia spp. & 15 & & \\
\hline \multicolumn{4}{|l|}{ G ros intestin } \\
\hline O esophagostomum radiatum & 35,5 & 114 & $1-1200$ \\
\hline Trichuris spp. & 1,6 & 25 & \\
\hline \multicolumn{4}{|l|}{ Autres } \\
\hline Schistosoma spp. & 1,6 & & \\
\hline Setaria labiatopapillosa & 30,6 & & \\
\hline O ocystes de coccidies & 9 & 350 & $200-600$ \\
\hline Trypanosoma spp. & 11,2 & & \\
\hline Microfilaires & 24,1 & & \\
\hline Babesia spp. & 3,6 & & \\
\hline
\end{tabular}
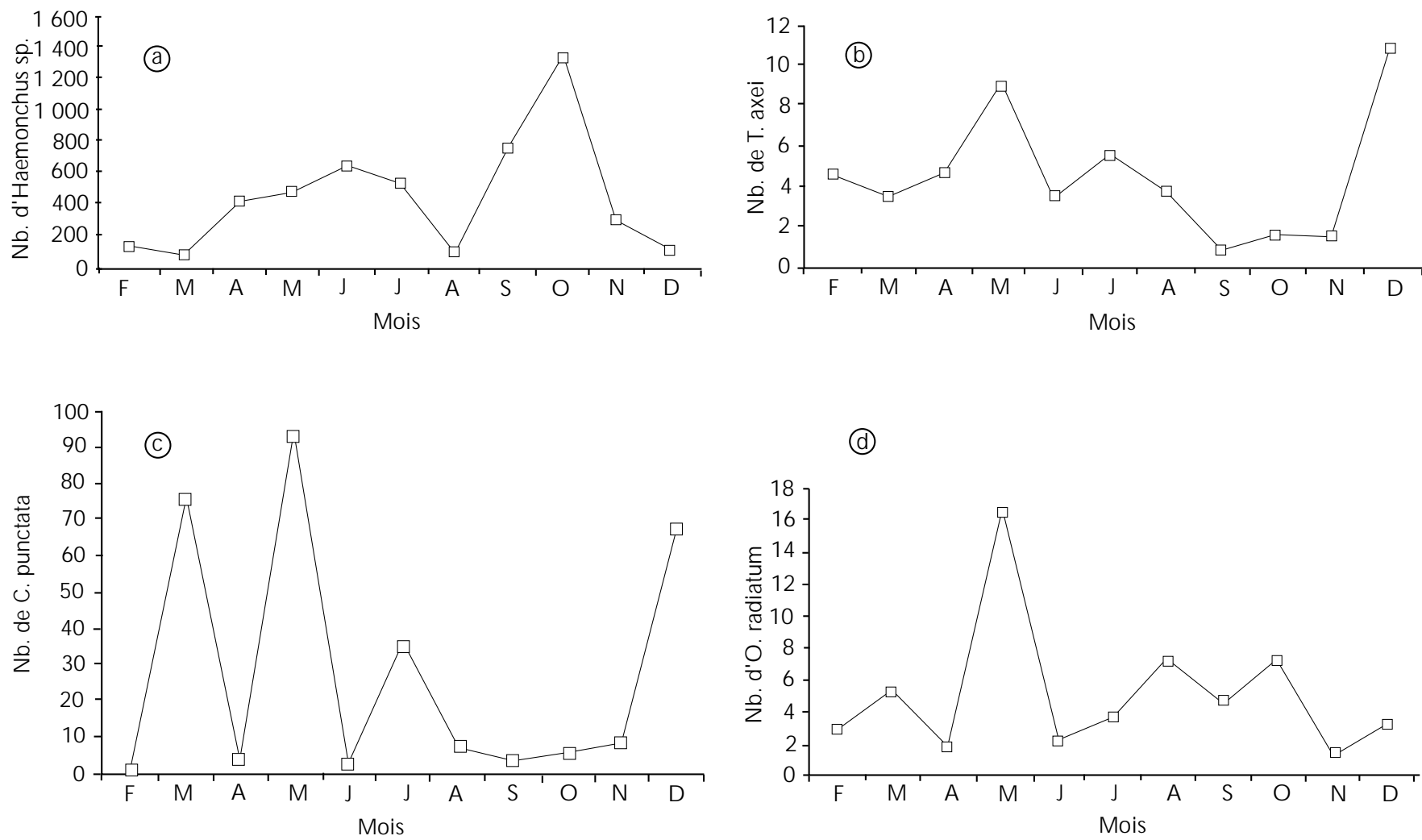

Figure 2 : moyennes géométriques des intensités mensuelles de Haemonchus sp. (a), T. axei (b), C. punctata (c) et 0 . radiatum (d) chez les bovins de la zone centre de savane humide de la Côte d'Ivoire. 


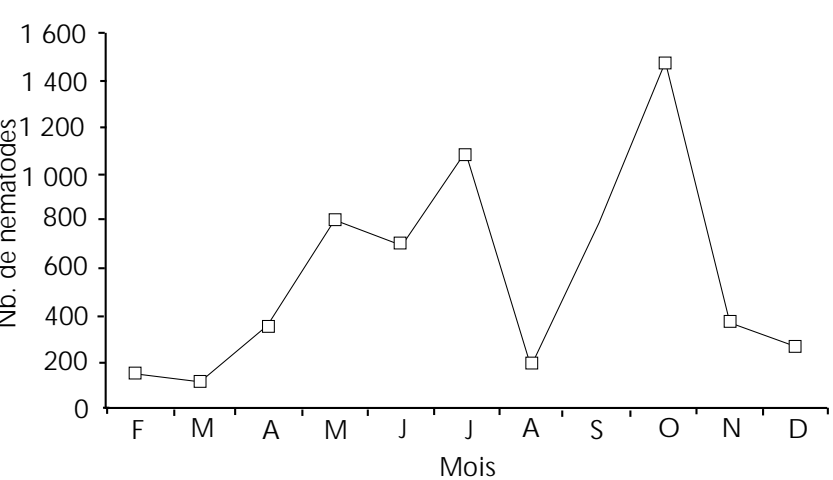

Figure 3 : moyennes géométriques des intensités mensuelles de tous les nématodes gastro-intestinaux chez les bovins de la zone centre de savane humide de la Côte d'Ivoire.
Les jeunes animaux avaient des intensités parasitaires (tableau II) et un opg significativement plus élevés $(\mathrm{p}<0,03)$ que les adultes âgés de plus de trois ans. La diversité parasitaire était également plus grande chez les jeunes. Ainsi, tandis que 65 p. 100 des sujets âgés de moins de trois ans hébergeaient 4 à 6 espèces de nématodes, seuls 5 p. 100 des adultes en hébergeaient autant.

Les prévalences des autres parasites rencontrés au cours de cette étude étaient de 30,6 p. 100 pour Setaria labiatopapillosa, 24,1 p. 100 pour les microfilaires, 11,2 p. 100 pour les trypanosomes (Trypanosoma brucei, T. congolense, T. vivax), 3,6 p. 100 pour Babesia sp. et 1,6 p. 100 pour Schistosoma sp.

\section{DISCUSSION}

La présente étude met en évidence un total de 14 espèces ou genres de parasites, incluant six espèces de nématodes gastrointestinaux, chez les bovins de race N'Dama du centre de la Côte d'Ivoire. Cet inventaire est proche de celui rapporté pour d'autres régions de l'Afrique de l'Ouest, telles que la Guinée (1), la Gambie $(3,11)$, le Sénégal (10), le Burkina Faso (12) et le Mali (13). Dans ces pays, d'autres espèces ont également été observées : Bunostomum phlebotomum, Strongyloides papillosus, T. colubriformis et $C$. curticei. En Côte d'Ivoire, comme ailleurs en Afrique de l'Ouest, Haemonchus sp. et Cooperia spp. étaient les parasites les plus importants.

La présence continue des infections parasitaires est certainement due aux conditions climatiques favorables (figure 1) pour le développement et la survie des larves infestantes des nématodes gastrointestinaux. Les variations saisonnières des intensités parasitaires et des excrétions d'œufs, bien que non statistiquement significatives pour certaines, ont montré des pics, la plupart du temps en mai et en septembre-octobre. Ces pics correspondent approximativement aux périodes des saisons des pluies, favorables au développement et à la transmission aux bovins sur les pâturages des larves infestantes.

Bien que l'incubation de la muqueuse abomasale dans un liquide de digestion pendant $12 \mathrm{~h}$ ait pu provoquer la lyse de larves, l'absence complète de larves Haemonchus sp. dans le digestat

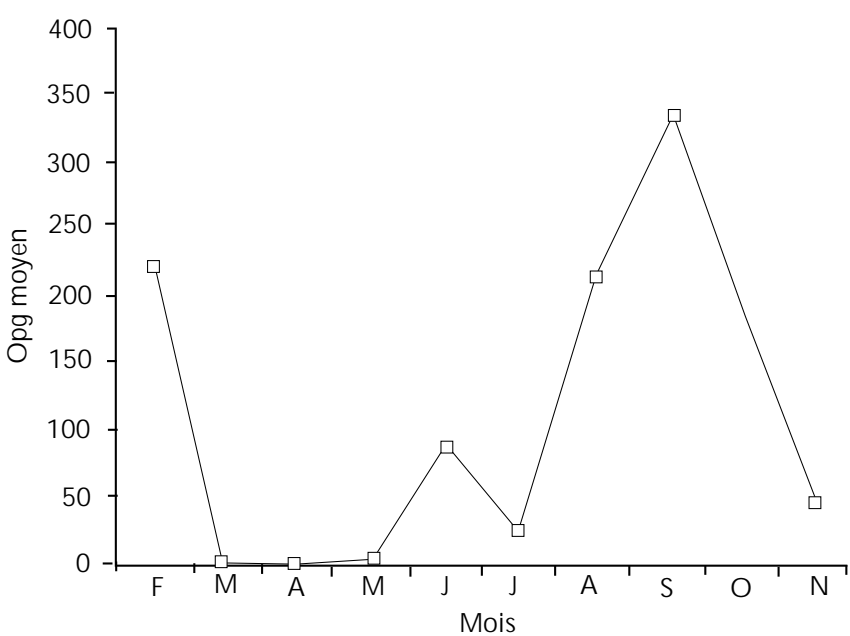

Figure 4 : moyennes géométriques du nombre d'œufs de strongles par gramme de fèces (opg) chez les bovins de la zone centre de savane humide de la Côte d'Ivoire.

\section{Tableau II}

Fréquence des intensités des nématodes gastro-intestinaux dans deux classes d'âge de bovins de la zone centre de savane humide de la Côte d'Ivoire

\begin{tabular}{lcc} 
Intensités & \multicolumn{2}{c}{ Fréquence (\%) } \\
\cline { 2 - 3 } & $<\mathbf{3}$ ans & $>\mathbf{3}$ ans \\
\hline 1000 & 46 & 74 \\
$1001-2000$ & 27 & 21 \\
$2001-3000$ & 9 & 2,5
\end{tabular}

laisse à penser que l'hypobiose larvaire n'existe pas dans le centre de la Côte d'Ivoire. Des résultats similaires ont été obtenus chez les ovins au sud (4), aussi bien qu'au centre (observations non publiées) de la Côte d'Ivoire, et chez les bovins, les ovins et les caprins en Guinée (1). Dans les pays d'Afrique de l'Ouest tels que la Gambie $(3,11)$ et le Sénégal $(10)$, où la saison sèche est longue et bien marquée, l'hypobiose constitue le principal mode de survie des nématodes gastro-intestinaux durant cette saison défavorable. En Côte d'Ivoire, le climat tropical humide étant favorable au développement des larves infestantes durant toute l'année, les nématodes n'ont pas la nécessité biologique d'entrer en hypobiose.

La baisse des intensités parasitaires chez les animaux de plus de trois ans est l'indication d'une résistance naturelle observée également chez les bovins N'Dama en Gambie $(3,14)$. Il aurait été intéressant d'examiner des animaux plus jeunes, mais ceux-ci ne sont que rarement abattus.

Malgré le climat tropical humide du centre de la Côte d'Ivoire, favorable au développement et à la survie des larves infestantes des nématodes gastro-intestinaux, les intensités parasitaires étaient relativement faibles. Cela peut être dû au système d'élevage extensif pratiqué dans cette région et à la faible pression parasitaire qui en résulte. 


\section{CONCLUSION}

Malgré certains biais comme le nombre inégal des animaux jeunes et âgés et des mâles et des femelles, ce travail permet de tirer quelques conclusions intéressantes. Dans la zone centre de la Côte d'Ivoire, les infections parasitaires sont survenues tout au long de l'année, avec des pics au cours des deux saisons des pluies. Les animaux âgés de moins de trois ans ont été les plus sensibles au parasitisme. Ils devraient être les seuls à bénéficier de traitements anthelminthiques (14). Ces traitements pourraient être effectués deux fois par an, en avril et en septembre pour éliminer les pics parasitaires. Une analyse coût/bénéfice devrait toutefois être faite avant de mettre en place tout programme de traitement stratégique. Pour cela, une étude sur un nombre plus important d'animaux serait souhaitable.

\section{Remerciements}

Nous exprimons nos remerciements à l'Académie suisse des sciences à Berne, Suisse, pour son appui financier et au Centre suisse de recherches scientifiques à Abidjan, Côte d'Ivoire, pour son assistance technique dans la réalisation de ce travail.

\section{BIBLIO G RAPHIE}

1. ANKERS P., FOFANA S., BIAYE A., 1997. Les dominantes du parasitisme helminthique chez les bovins, ovins et caprins en Guinée maritime, République de Guinée. Revue Elev. Méd. vét. Pays trop., 50 : 111-116.

2. GORDON H.M., WHITLOCK H.V., 1939. A new technique for counting nematode eggs in sheep faeces. J. Counc. Sci. Ind. Res. Aust., 10: $50-52$.

3. KAUFMANN J., PFISTER K., 1990. The seasonal epidemiology of gastrointestinal nematodes in N'Dama cattle in The Gambia. Vet. Parasitol., 37: 45-54.

4. KOMOIN-OKA C., ZINSSTAG J., PANDEY V.S., FO FANA F., N'DEPO A., 1999. Epidémiologie des parasites des ovins de la zone sud forestière de la Côte d'Ivoire. Revue Elev. Méd. vét. Pays trop., 52 : 39-46.

5. Manual of veterinary parasitological laboratory techniques, 1986. Reference book 418, 3rd ed. London, UK, Ministry of Agriculture, Fisheries and Food, Her Majesty's Stationary 0 ffice, $160 \mathrm{p}$.

6. MATTIOLI R.C., BAH M., FAYE J., KORA S., CASSAMA M., 1993. A comparison of field tick infestation on N'Dama, Gobra zebu and N'D ama x Gobra crossbred cattle. Vet. Parasitol., 47: 139-148.

7. MATTIOLI R.C., CASSAMA M., KORA S., 1997. A comparative study of gastrointestinal nematode output in N'Dama, zebu and N'Dama $x$ zebu crossbred cattle. Parasitologia, 34: 109-113.

8. MATTIOLI R.C., JAITNER J., CLIFFORD D.J., PANDEY V.S., VERHULST A., 1998. Trypanosome infections and tick infestations: susceptibility in N'Dama, Gobra zebu and Gobra x N'Dama crossbred cattle exposed to natural challenge and maintained under high and low surveillance of trypanosome infections. Acta trop., 71: 57-71.
9. MURRAY M., MURRAY P.K., M CINTYRE W.I.M., 1977. An improved parasitological technique for diagnosis of African trypanosomiasis. Trans. R. Soc. trop. Med. Hyg., 71: 325-326.

10. NDAO M., BELOT J., ZINSSTAG J., PFISTER K., 1995. Epidémiologie des nématodoses gastro-intestinales des bovins dans la zone sylvo-pastorale au Sénégal. Revue M éd. vét., 146 : 129-134.

11. NDAO M., PANDEY V.S., ZINSSTAG J., PFISTER K., 1995. Helminth parasites and hypobiosis of nematodes in N'Dama cattle during the dry season in The Gambia. Vet. Parasitol., 60: 161-166.

12. OUEDRAOGO A., OUATTARA L., KAUFMANN J., PFISTER K., 1992. Epidémiologie des nématodes gastro-intestinaux des ruminants au Burkina Faso : spectre, fréquences et variations saisonnières. In : $7^{\mathrm{e}}$ Conf. AIMVT, Yamoussoukro, Côte d'Ivoire, 14-18 septembre 1992, p. $749-750$.

13. TEMBELY S., 1986. An abattoir survey of gastrointestinal helminth parasites in cattle, sheep and goats in Mali (West Africa). PhD Dissertation, Graduate College of Texas, A\&M University, College Station, TX, USA, $116 p$.

14. ZINSSTAG J., ANKERS P., DEMPFLE L., NJIE M., KAUFMANN J., ITTY P., PFISTER K., PANDEY V.S., 1997. Effect of strategic gastrointestinal nematode control on growth of N'Dama in Gambia. Vet. Parasitol., 68: 143-153.

15. ZINSSTAG J., ANKERS P., NDAO M., BONFOH B., PFISTER K., 1998. Multiparasitism, production and economics in domestic animals in sub-Saharan W est Africa. Parasitology Today, 14: 46-49.

Reçu le 09.05.2000, accepté le 08.11 .2000 


\section{Summary}

Komoin-O ka C., Zinsstag J., Fofana F., N'D epo A., Pandey V.S. Epidemiology of gastrointestinal nematodes of cattle in Central Côte d'Ivoire

From February to December 1997, six head of N'Dama cattle originating from the Central zone of humid savanna in Côte d'Ivoire were examined postmortem monthly. Fourteen species/genera of parasites were identified: 8 nematodes, 2 trematodes, 1 cestode and 3 protozoa. All animals were infected with at least one of the following gastrointestinal nematodes: Haemonchus sp. (95\%), Cooperia punctata (50\%), O esophagostomum radiatum (35.5\%), Trichostrongylus axei $(30 \%)$, Cooperia pectinata $(8.1 \%)$ and Trichuris sp. (1.6\%). Setaria labiatopapillosa was found in $30.6 \%$ of the animals examined, microfilaria in $24.1 \%$, paramphistomes in $80.6 \%$ and Moniezia sp. in 15\%. Trypanosomes and Babesia spp. were found in 11.2 and $3.6 \%$ of the animals, respectively. The egg counts and worm burden of gastrointestinal nematodes varied with peaks in May and September/O ctober, i.e. during the rainy seasons. Young animals $1-3$ years of age had higher worm burdens than animals over 3 years old $(P<0.05)$. No larval hypobiosis was detected. In spite of a favorable humid climate, the worm burden of this N'Dama cattle originating from an extensive husbandry system was relatively low. Anthelmintic treatments appear necessary in younger animals only.

Key words: N'Dama cattle - Helminth - Nematoda Epidemiology - Côte d'Ivoire.

\section{Resumen}

Komoin-O ka C., Zinsstag J., Fofana F., N'Depo A., Pandey V.S. Epidemiología de los nemátodos gastrointestinales de los bovinos en la región central de Costa de Marfil

Entre febrero y diciembre de 1997, se autopsiaron cada mes seis bovinos de la raza N'Dama, provenientes de la zona central de sabana húmeda de la Costa de Marfil. Se encontraron 14 especies o géneros de parásitos: 8 nemátodos, 2 tremátodos, 1 céstodo y 3 protozoarios. Todos los animales estaban infestados por al menos una de las siguientes especies de nemátodos gastrointestinales: H aemonchus sp. (95\%), Cooperia punctata ( $50 \%)$, O esophagostomum radiatum (35,5\%), Trichostrongylus axei $(30 \%)$, Cooperia pectinata $(8,1 \%)$ y Trichuris sp. (1,6\%). Setaria labiatopapillosa fue encontrado en $30,6 \%$ de los animales, microfilarias en $24,1 \%$, paranfistomos en $80,6 \%$ y Monezia sp. en $15 \%$. Los tripanosomas y Babesia spp. fueron encontrados en 11,2 y $3,6 \%$ de los animales, respectivamente. Las excreciones de huevos y las intensidades parasitarias de los nemátodos gastrointestinales mostraron fluctuaciones, con picos en mayo y en septiembre-octubre, periodos correspondientes a las estaciones de lluvias. Los animales de 1-3 años de edad presentaron intensidades parasitarias más elevadas que los mayores de 3 años $(P<0,05)$. No se detectó ninguna hipobiosis larval. A pesar de las condiciones climáticas húmedas favorables, las intensidades parasitarias de estos bovinos de raza N'Dama, provenientes de sistemas de crianza de tipo extensivo, fueron relativamente bajas. Solo se justifican entonces tratamientos antihelmínticos en los animales jóvenes.

Palabras clave: Ganado bovino N'Dama - Helminto - Nematoda - Epidemiología - Côte d'Ivoire. 